\title{
Structural Response of Human Serum Albumin to Oxidation: Biological Buffer to Local Formation of Hypochlorite
}

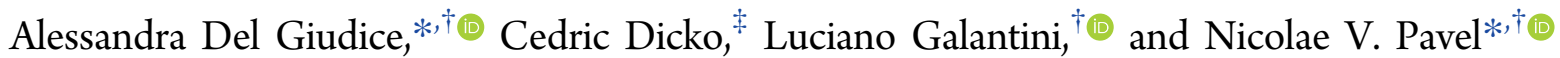 \\ ${ }^{\dagger}$ Department of Chemistry, Sapienza University of Rome, P. le A. Moro 5, 00185 Rome, Italy \\ ${ }^{\ddagger}$ Department of Chemistry, Division for Pure and Applied Biochemistry, Lund University, Naturvetarvägen 14, 2241 Lund, Sweden
}

Supporting Information

ABSTRACT: The most abundant plasma protein, human serum albumin (HSA), plays a key part in the body's antioxidant defense against reactive species. This study was aimed at correlating oxidant-induced chemical and structural effects on HSA. Despite the chemical modification induced by the oxidant hypochlorite, the native shape is preserved up to oxidant/HSA molar ratio $<80$, above which a structural transition occurs in the critical range 80-120. This conformational variation involves the drifting of one of the end-domains from the rest of the protein and corresponds to the loss of one-third of the $\alpha$-helix and a net increase of the protein negative charge. The transition is highly reproducible suggesting that it represents a well-defined structural response typical of this multidomain protein. The ability to tolerate high levels of chemical modification in a folded or only partially unfolded state, as well as the stability to aggregation, provides albumin with optimal features as a biological buffer for the local formation of oxidants.

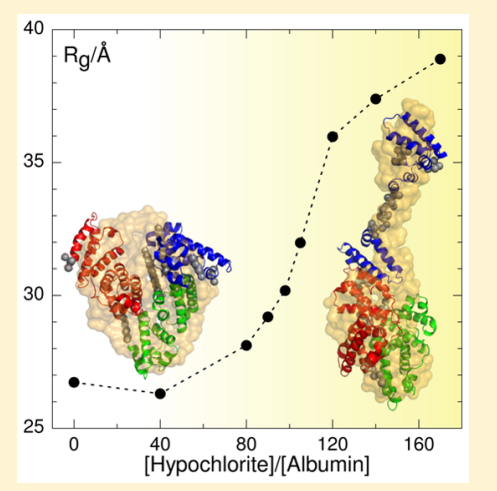

\section{INTRODUCTION}

Human serum albumin (HSA) is the most abundant protein contained in plasma, and is also found in tissues and secretions throughout the body. It is produced in the liver and contributes to maintaining osmotic pressure in blood. Moreover, it binds nutrients, metabolites, and drugs in order to transport and release them. ${ }^{1,2}$ The albumin structure ${ }^{3}$ is characterized by a repeating pattern of three homologous domains (I, II, III), in turn composed of three loops of $\alpha$-helices, each stabilized by the presence of intraloop disulfide bridges. This carrier protein is also an obvious target of extracellular reactive oxidant species due to its high abundance in plasma. It is for this reason considered the main antioxidant defense in blood. ${ }^{4-6}$

One of the main oxidants released in the extracellular environment is hypochlorite, a key component of the inflammatory response. ${ }^{7}$ It has bactericidal activity but it has become apparent that collateral damage to host tissues is a key event in a number of human pathologies linked with inflammation. $^{8-10}$ Rate constants ${ }^{11,12}$ for reaction of hypochlorite with individual biological components have shown that proteins are by far the major targets for hypochlorite in plasma, ${ }^{13}$ and HSA, as the most abundant protein, is thought to be the most probable target in general. Oxidatively modified forms of HSA have indeed been detected in several pathological conditions characterized by oxidative stress, ${ }^{14}$ and the characterization of model systems of oxidized HSA has mainly been addressed through chemical essays or mass spectrometry methods to quantify and identify chemical modifications induced by hypochlorite, ${ }^{13,15-17}$ as well as by detecting spectral variations in UV absorption and fluorescence spectroscopy. $^{18-20}$
The HSA response to oxidation constitutes a complex picture as the extent of protein modification induced by oxidants widely changes depending on the oxidant-to-protein molar ratio and time. It is therefore difficult to create a complete image of the HSA hypochlorite-induced damage from the existing literature. Some interesting systematic studies have been reported by Pattison et al. ${ }^{13}$ on the homologue bovine serum albumin (BSA) clarifying the effect on the amino acidic composition of increasing equivalents of the oxidant hypochlorite.

It is foreseeable that repeated exposure of HSA to oxidative environments at high oxidant protein molar ratios could significantly affect its biological activity. With knowledge of the well-established strict dependence of protein functionality on protein structure, a correlation between the structure and the oxidation state of HSA is expected to be crucial for rationalizing the effect of oxidation on the functional ability of this protein. This study will help clarify the mechanism of oxidant defense played by HSA and explain whether structural features make this protein particularly suitable as a reactive species scavenger.

To address these questions, we correlated chemical damage and protein structural stability over a broad range of oxidant/ protein ratios $(\mathrm{OPR}-[\mathrm{NaOCl}] /[\mathrm{HSA}]$, ranging from 10 to $170)$, comprising both relevant physiological oxidant concentrations (high-micromolar or low-millimolar levels in inflamed tissues $^{21}$ ) and higher oxidant/protein molar excesses explored in in vitro experiments. We used a new multitechnique instrumental platform to probe both chemical and structural

Received: August 25, 2016

Revised: November 8, 2016

Published: November 10, 2016 
changes. $^{22}$ It combined the simultaneous collection of smallangle X-ray scattering (SAXS), UV/vis absorbance spectra, and fluorescence emission on the same sample volume and it is in the following referred to as SUrF (SAXS-UV/vis and fluorescence). The reliability of the structural characterization of the oxidized protein in solution was enhanced by the in-line separation with size exclusion chromatography (SEC).

The results demonstrated that the high resistance of the HSA functionality to oxidation is correlated to a stability of its tertiary structure up to relevant oxidation conditions (oxidant/ protein molar ratio <80). Under further oxidative stress, HSA partially loses its native tertiary and secondary structure, and assumes an elongated form. The structural transition occurs very reproducibly in the critical range of an oxidant/HSA molar ratio between 80 and 120 . This behavior illustrates a new feature from the known conformational plasticity of HSA in conditions that destabilize its native folding. ${ }^{2}$

\section{EXPERIMENTAL SECTION}

Sample Preparation. Human serum albumin (HSA) lyophilized powder, fatty acid- and globulin-free ( $\geq 99 \%$, type A3782), obtained from Sigma was dissolved in $10-\mathrm{mM}$ phosphate buffer at $\mathrm{pH} 7.4$ and centrifuged at $18 \times 10^{3} \mathrm{~g}$ for $10 \mathrm{~min}$ at $4{ }^{\circ} \mathrm{C}$. The protein concentration was measured by collecting a UV spectrum of the stock solution with a $1 \mathrm{~mm}$ path length, using the absorbance at $280 \mathrm{~nm}$ and the extinction coefficient ${ }^{23} 37500 \mathrm{M}^{-1} \mathrm{~cm}^{-1}$.

Mixing conditions constitute an important aspect of rapid chemical reactions. Here, we optimized the method of preparation to minimize inhomogeneity during mixing and could thus avoid the uncontrolled and exclusively local exposure of the protein to higher concentrations of oxidant. We used SEC as a guide and assumed that if the chromatograms of the oxidized HSA samples showed the expected ${ }^{24,25}$ two peaks with the monomer/oligomer relative populations of a native sample (about 85 and 15\%), then the mixing conditions ensured homogeneity.

The NaOCl-oxidized HSA samples were prepared by mixing equal volumes of $\mathrm{HSA}$ and $\mathrm{NaOCl}$ stock solutions. In the SUrF "single-shot" experiments (see Supporting Information Section $1)$, the final protein concentration was about $5 \mathrm{~g} / \mathrm{L}(75 \mu \mathrm{M})$, while it reached about $10 \mathrm{~g} / \mathrm{L}(150 \mu \mathrm{M})$ in the samples injected in the SEC column. The HSA concentration in the oxidized and then desalted samples which underwent zeta-potential measurements was around $30 \mu \mathrm{M}$. In the case of $\mathrm{CD}$ measurements the final protein concentration was $22.75 \mu \mathrm{M}$. Samples with oxidant/protein ratios $([\mathrm{NaOCl}] /[\mathrm{HSA}])$ from 0 up to 170 were analyzed. All the experiments, unless otherwise specified, were performed in degassed 10-mM phosphate buffer with $\mathrm{pH} 7.4$, at room temperature $\left(24 \pm 1{ }^{\circ} \mathrm{C}\right)$. After preparation, the oxidized protein samples were also stored at room temperature.

A series of fresh $\mathrm{NaOCl}$ working solutions with the concentration required to reach the desired oxidant/protein ratio in the final sample were used in the experiments. First, a stock solution of $\mathrm{NaOCl}$ was prepared by diluting commercial $\mathrm{NaOCl}$ solution (from Sigma, type 425055, reagent grade, available chlorine $10-15 \%$ ) with buffer. The concentration of this $\mathrm{NaOCl}$ stock solution (maximum $80 \mathrm{mM}$ ) was determined by measuring the UV absorbance at $293 \mathrm{~nm}$ after dilution in a $\mathrm{NaOH}$ solution with $\mathrm{pH} 12$, in a $1 \mathrm{~cm}$ quartz cuvette, using the molar extinction coefficient ${ }^{26}$ value of $350 \mathrm{M}^{-1} \mathrm{~cm}^{-1}$. The working solutions for the oxidation of HSA were then obtained by conveniently diluting the stock solution in buffer. The working solutions of $\mathrm{NaOCl}$ were stored on ice or in the refrigerator at $4{ }^{\circ} \mathrm{C}$ during the day of the experiments and brought to room temperature before performing the mixing with the protein solution.

Due to the alkalinity of the commercial initial solution of $\mathrm{NaOCl}$ and the low concentration of the buffer employed, the HSA samples prepared at highest protein concentration and highest oxidant/protein ratio could reach a $\mathrm{pH}$ as high as 9 just after mixing. However, the results of structural characterization refer to the oxidized protein eluted with buffer in a SEC experiment and so equilibrated at native $\mathrm{pH}$ (7.4). SAXS characterization was performed on the oxidized samples also without SEC and the agreement between the results of the two approaches is discussed in the Results Section. In the samples for $\mathrm{CD}$ measurements prepared at lower protein concentration the highest $\mathrm{pH}$ reachable just after the mixing of HSA and $\mathrm{NaOCl}$ solutions was 8 , but decayed to less than 7.7 in $5 \mathrm{~h}$.

The time elapsed between the mixing of HSA and $\mathrm{NaOCl}$ and the data acquisition in the case of the SUrF experiments is specified in the Supporting Information, Section 1. In the case of the $\mathrm{CD}$ experiments, the data acquisition started between 15 and $30 \mathrm{~min}$ after the mixing; the measurements were also repeated after $18 \mathrm{~h}$ from preparation and no variation of the CD spectra of the samples was observed. In order to measure the zeta potential of oxidized HSA by means of electrophoresis, the samples were prepared as previously described and then desalted in phosphate buffer with a GE-healthcare PD-G25 Miditrap buffer-exchange column, 10 min after oxidation.

Characterization with the SUrF Platform. The combined SAXS and spectroscopic experiments were performed at the I-911 beamline at MAXlab, for which the specifications were reported by Labrador et al. ${ }^{27}$ An outline of the SUrF multi probe set up was reported by Haas et al. ${ }^{22}$ We used the second generation of the platform and a detailed description of the experimental procedures used in data acquisition and treatment is reported in the Supporting Information Section 1.

Circular Dichroism. The circular dichroism (CD) spectra were recorded between 260 and $190 \mathrm{~nm}$ in a JASCO J-750 spectropolarimeter at $25{ }^{\circ} \mathrm{C}$ using a bandwidth of $0.5 \mathrm{~nm}$, a 50 $\mathrm{nm} / \mathrm{min}$ scan rate and averaging 3 acquisitions. The demountable quartz cuvette path length was $0.1 \mathrm{~mm}$ and the protein concentration was $22.75 \mu \mathrm{M}$. The raw data in millidegrees were subtracted by the buffer contribution and converted into medium residue ellipticity (MRE, units [deg $\left.\mathrm{cm}^{2} \mathrm{dmol}^{-1}\right]$ ) considering that HSA has 585 amino acids. The MRE at $222 \mathrm{~nm}$ was used to estimate the $\alpha$-helix secondary structure content, according to the method suggested by Chen et al. $^{28}\left(\% \alpha\right.$-helix $\left.=100 \times\left(-\mathrm{MRE}_{222 \mathrm{~nm}}-2340\right) / 30300\right)$. In this case error bars were computed estimating the standard deviation of the MRE values dependent on a $1 \%$ uncertainty of the protein concentration value, a $0.5 \%$ uncertainty of the demountable cell path length and a $1 \%$ uncertainty due to noise in the spectra. In addition, the full CD spectra between 190 and $250 \mathrm{~nm}$ were analyzed by means of both the BeStSel ${ }^{29} \mathrm{Web}$ server and the CONTIN/LL algorithm of the software package CDpro. ${ }^{30}$ Both methods provide a least-squares fit of the farUV CD spectra in terms of secondary structure components (e.g., $\alpha$-helix, $\beta$-strands, turns, disordered/other). In the case of HSA, the $\alpha$-helical (both regular and distorted at the ends of the segments) and disordered/turn structures accounted for most $(>93 \%)$ of the secondary structure predicted in all conditions. 

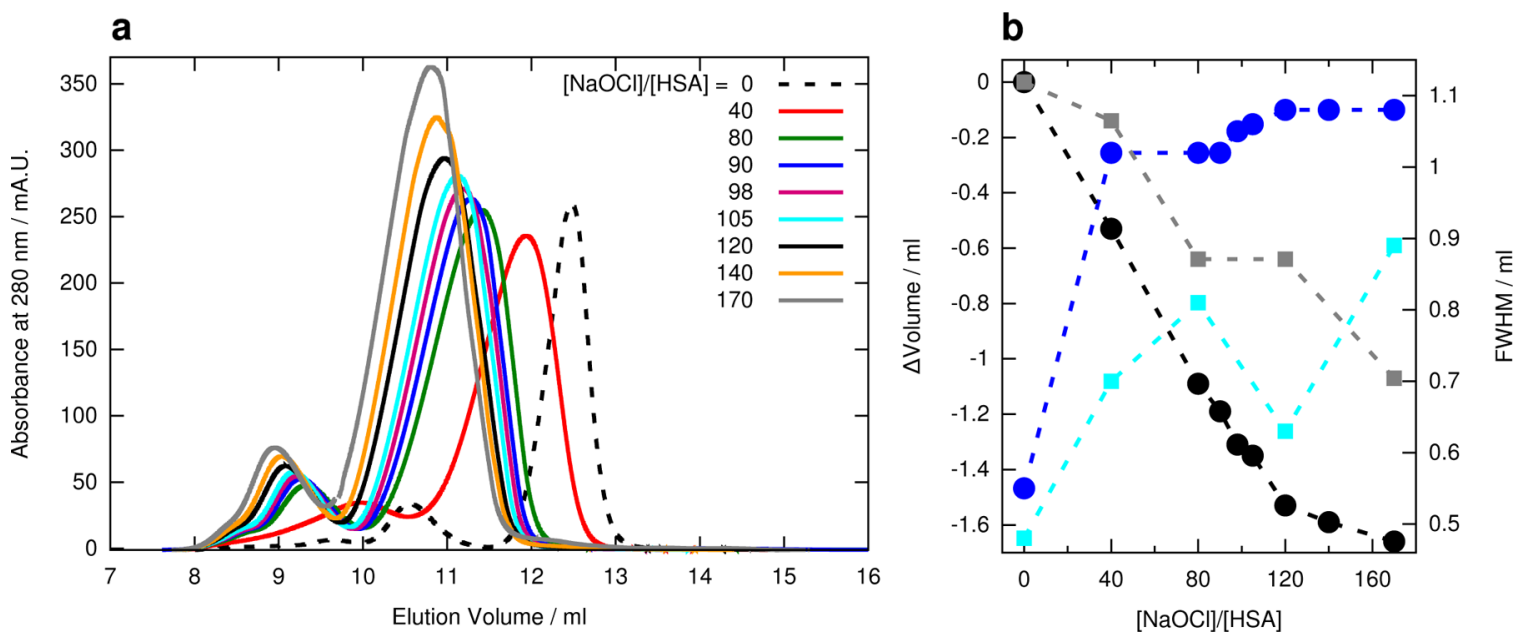

Figure 1. SEC analysis of increasingly oxidized HSA samples showed a systematic shift of the retention volume and peak broadening. (a) Chromatogram traces recorded by the UV-280 nm detector of the SEC apparatus during the elution of oxidized HSA samples with oxidant/protein ratios: 0 (black dotted), 40 (red solid), 80 (green solid), 90 (blue solid), 98 (purple solid), 105 (cyan solid), 120 (black solid), 140 (orange solid), 170 (gray solid). (b) Relative decrease of the elution volume compared with the reference sample ( $\Delta$ Volume, ordinate axis to the left, black dots) and increase of full width at half-maximum (FWHM, to the right, blue dots) of the main elution peak. The increase of the ionic strength of the elution buffer (containing 0.15- $\mathrm{M} \mathrm{NaCl}$ ) determined smaller volume shifts (gray squares) and peak broadening (cyan squares).

Zeta Potential. The measurements were performed at 25.0 ${ }^{\circ} \mathrm{C}$, using a MALVERN NanoZetaSizer apparatus (Malvern Instruments LTD, UK) equipped with a $5 \mathrm{~mW} \mathrm{HeNe}$ laser. This instrument technique combines laser doppler velocimetry and phase analysis light scattering to determine the average mobility and the mobility distribution. The $\zeta$-potential of the particles (in $\mathrm{mV}$ ) was obtained from the electrophoretic mobility $u$ measured using the Smoluchowski relation $\zeta=u \eta / \varepsilon$, where $\eta$ and $\varepsilon$ are the viscosity and the permittivity of the solvent phase (water), respectively. The measurements were performed using a folded capillary cell (Malvern, UK), and the runs were carried out in triplicate for each sample, each repeat consisting of 12 to 24 sub-runs. The reported values are the average of the three replicate measurements on the same samples, and the error bars represent the standard deviation.

SAXS Data Analysis. SAXS data reduction (radial averaging, buffer subtraction) was performed with the software RAW, ${ }^{31}$ using silver behenate to calibrate the range of the scattering vector $(q)$ and water to convert the detector data in absolute intensity. ${ }^{32,33}$ The model-independent analysis of the scattering profiles in the approximation of the monodisperse ideal solution gives information on the overall shape and dimensions of the particles. A first estimate of the radius of gyration $\left(R_{\mathrm{g}}\right)$ according to the Guinier approximation ${ }^{34}$ at low $q\left(I(q)=I(0) \cdot \exp \left[\frac{-\left(q R_{\mathrm{g}}\right)^{2}}{3}\right]\right)$ was made by fitting the data with PRIMUS, ${ }^{35}$ considering a $q$ range between $q_{\min }=0.012 \AA^{-1}$ and $q_{\max }=1.3 / R_{\mathrm{g}}$. Model-free analysis through indirect Fourier transformation, providing the pair distance distribution function $(P(R))$, from which the maximum particle distance $\left(D_{\max }\right)$, and the direct space $R_{\mathrm{g}}$ and scattering intensity at zero angle $\left(I_{0}\right)$ could be calculated, was performed with $\mathrm{GNOM}^{36}$ and IFT-BayesApp. ${ }^{37}$ The same $q$ range $\left(0.012-0.35 \AA^{-1}\right)$ was selected for all data. IFT-BayesApp also gave the equivalent ellipsoid axis ratio ${ }^{38}$ that is very diagnostic of a transition from a globular to elongated shape.

The $a b$ initio program GASBOR ${ }^{39}$ was used in order to build 3D-models from the SAXS profiles. This software employs the average scattering factor of amino acids and makes it possible to model the particle as a protein chain-like assembly of dummyresidues, whose number is constrained according to the known sequence of the protein. The calculations were performed with P1 symmetry. A series of 20 models was generated, and the similarity of the obtained structures was verified by DAMAVER $^{40}$ and DAMCLUST, ${ }^{35}$ in which the superposition was performed by the SUPCOMB ${ }^{41}$ code. A normalized spatial discrepancy (NSD) value was calculated and used as a parameter to determine the difference between two threedimensional models. The model most similar to the others was chosen as representative.

The combined rigid-body/ab initio approach implemented in BUNCH $^{42}$ was also used to model possible partially unfolded conformations of HSA fitting the scattering data of the oxidized protein. High-resolution coordinates found in the pdb entry $1 \mathrm{AO}^{43}$ were employed to create the starting model to be optimized, which was composed of rigid moieties interconnected by flexible linkers.

\section{RESULTS}

Fingerprinting Oxidized HSA Substates by SEC-SAXS. The size exclusion chromatography (SEC) analysis of the reference nonoxidized sample showed as expected a highly monomeric protein solution with a small contribution of oligomers. We observed that all the oxidized samples preserved the same monomer/oligomer relative populations as the native protein solution (Figure 1a). The oxidized samples showed a peak broadening and a systematic shift of the elution profile toward lower elution volumes compared with the reference sample. Values for the decrease of the elution volume of the main peak relatively to the reference sample are shown in Figure $1 b$ as a function of increasing OPR, together with the absolute increase of the full width at half-maximum. In principle the elution should take place in conditions of absence of interactions between protein and stationary phase. However, interactions of electrostatic nature could also contribute to the observed peak position and peak broadening, together with the determinant hydrodynamic properties of the protein and the existence of a population of conformers. In the case of the 

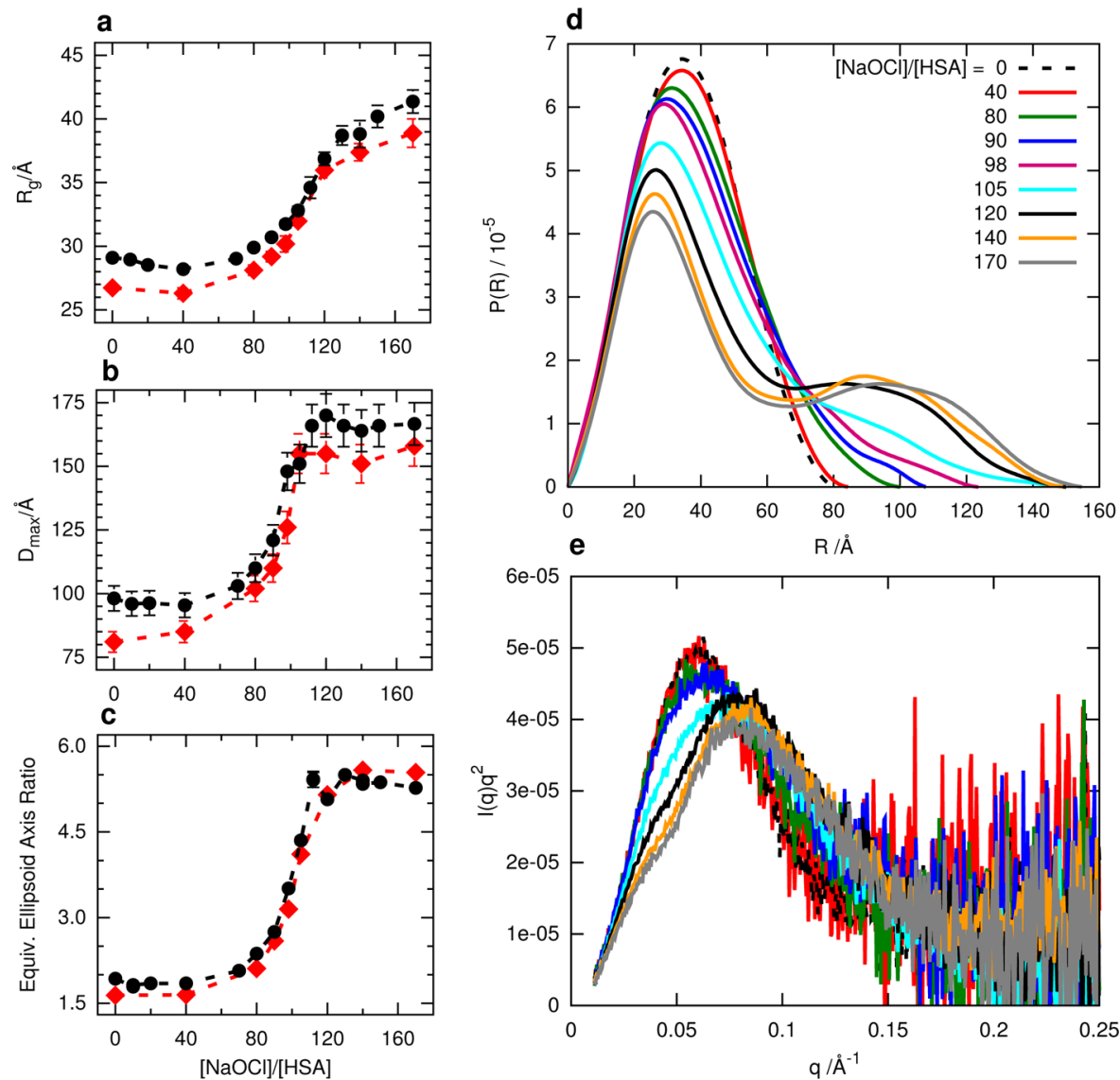

Figure 2. Variation of structural features derived from the SAXS data as a function of the oxidant/protein ratio $([\mathrm{NaOCl}] /[\mathrm{HSA}])$ suggests that the oxidized protein monomer assumes a progressively elongated shape. The parameters derived from the SEC-SAXS data collected at the monomer peak maximum (red) and from the "single shot" experiment without SEC (black) are compared. (a) Radius of gyration $\left(R_{\mathrm{g}}\right)$; the error bars show the standard deviation estimated by the fitting procedure. (b) Maximum intraparticle distance $\left(D_{\max }\right)$; error bars corresponding to $5 \%$ of the value are displayed. (c) Axis ratio of the equivalent ellipsoid of revolution. (d) Pair distance distribution functions derived from the SEC-SAXS data collected at the monomer peak maximum for increasingly oxidized HSA. (e) Representation of the scattered intensity $I(q)$ profiles collected at the monomer peak maximum for increasingly oxidized HSA in the form of Kratky plot $\left(I(q) \cdot q^{2}\right.$ vs $\left.q\right)$. This visualization helps to assess the degree of flexibility of proteins in solution since a bell-shape is expected for a globular particle while a plateau is expected for a coil.

oxidized protein samples, the oxidation degree could be correlated with an increase of electrostatic repulsion, which could be attenuated in the presence of an electrolyte. As shown in Figure $1 b$, the relative shift and the peak width were indeed reduced significantly when the ionic strength of the elution buffer was increased through the addition of $150 \mathrm{mM} \mathrm{NaCl}$.

The structural parameters obtained by the SAXS analysis of the monomer fraction had a sigmoidal dependence with increasing OPR (Figure 2). A steep increase of the total particle dimensions $\left(R_{\mathrm{g}}\right.$ and $D_{\max }$, Figure $\left.2 \mathrm{a}, \mathrm{b}\right)$ and axial ratio (Figure 2c) was observed at critical OPR values around 80, and a plateau was seen for values higher than 120 . The sigmoidal profile indicated that a transition between the low and high OPR values occurred, with the protein undergoing a structural rearrangement from its native globular shape to a more elongated one. The loss of the native tertiary structure was evidenced by a characteristic evolution of the $P(R)$ profiles from the typical bell shape of the native form to a bimodal distribution (Figure 2d).

In addition, the visualization of the scattering data in the form of a Kratky plot highlighted that this structural variation did not imply a significant increase of the protein chain flexibility that is typical of unfolded states. This representation (diagram of scattered intensity $I(q)$ multiplied by the squared scattering vector $q^{2}$ as a function of $q$ ) makes it possible to qualitatively distinguish a globular protein from a premolten globular structure or an advanced unfolded state. ${ }^{44}$ In the case of oxidized HSA when the OPR values increased, the initial bell shape of the Kratky plot demonstrated an increasing positive skewness, indicating a more elongated structure, ${ }^{45}$ but was far from reaching a plateau at higher $q$, typical for a protein behaving as a Gaussian-like coil (Figure 2e).

The oxidation-induced conformational change could be well depicted by comparing the low-resolution models obtained by fitting the experimental scattering profiles of the protein at increasing oxidative damage. In contrast with the hearth shape typical of the native form at low OPR values $(\leq 40)$, at high OPR values $(\geq 120)$ the representative models presented a markedly extended lobe (Figure 3 ). In the latter case, the average number of residues found in the extended lobe allowed us to roughly estimate that it was equivalent in size to one of the end-domains of HSA. An alternative modeling approach which assumes the known domain-structure of HSA and was used in the past to model the HSA unfolding in urea, ${ }^{46,47}$ was then tried to fit the same scattering data. The intact HSA domains II and III and the three loops of domain I were used as structural units interconnected by flexible linkers. Repeated optimizations converged to the same spatial distribution of 


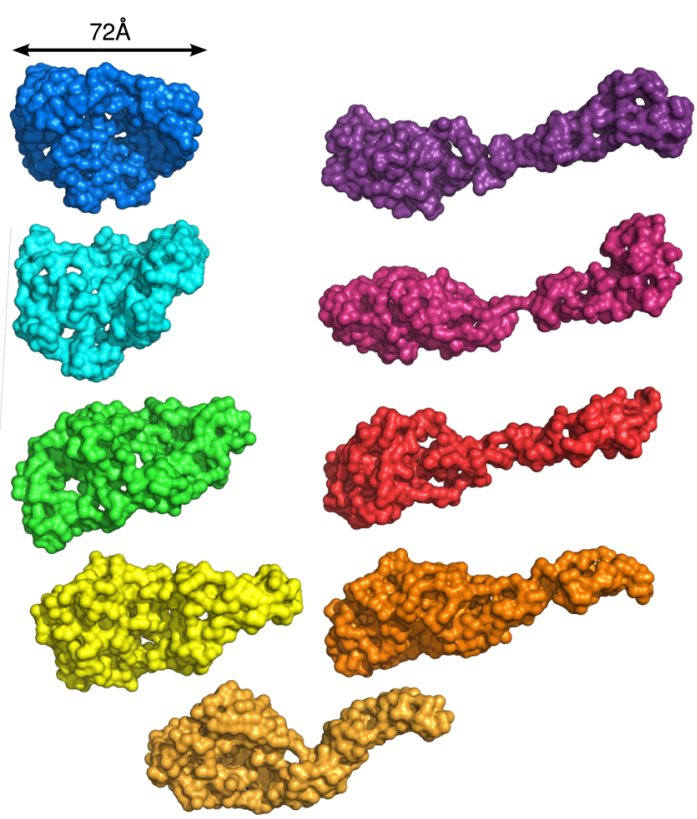

Figure 3. Low-resolution structural models of the increasingly oxidized HSA monomer derived from SAXS data fitting. Representative dummy atom models fitting SEC-SAXS data (from the maximum of the main elution peak) of oxidized HSA samples up to $0.35 \AA^{-1}$. Going from the top left to the top right the experimental $[\mathrm{NaOCl}] /[\mathrm{HSA}]$ ratios were: 0 (blue), 40 (cyan), 80 (green), 90 (yellow), 98 (light orange), 105 (orange), 120 (red), 140 (purple), 170 (violet). A dimensional bar corresponding to $72 \AA$ is shown as a reference.

domains, and supported the fact that a detachment of one of the end-domains of HSA induced by the increasing amount of hypochlorite chemical damage was compatible with the experimental data (Supporting Information, Figure S1).

The SAXS analysis for the series of NaOCl-treated HSA samples that did not undergo SEC (Supporting Information, Figure S2) showed the same behavior presented above, except for a slight incremental shift of the dimensional parameters (black dots in Figure 2a,b,c). This suggested that the contributions of HSA oligomers and also of possibly higher $\mathrm{pH}$ environment in the samples which underwent addition of the most concentrated $\mathrm{NaOCl}$ solutions were rather negligible in the process, and the intrinsic effect of the chemical modification caused by hypochlorite was the cause of the structural transition. The outcome was the same also when looking at the SAXS data collected at short times (between 5 and $15 \mathrm{~min}$ ) from the oxidant addition (Supporting Information, Figure S3b), demonstrating that the structural transformation induced by oxidation was fast and that, once in the highly oxidized state, albumin retained the open conformation and was not subject to aggregation or further degradation in this time frame. Measurements performed at longer times $(>24 \mathrm{~h})$ showed a slight decrease of the scattered intensity for the highly oxidized samples demonstrating a possible partial fragmentation ${ }^{48}$ of the protein. The phenomenon was modest and did not significantly affect the SAXS curve and $P(R)$ shapes (Supporting Information, Figure S4).

Secondary Structure and Surface Charge of the Oxidized HSA. Complementary experiments for the characterization of the secondary structure composition and average surface charge of the oxidized protein conformer were performed and the results are presented in Figure 4.

The far-UV circular dichroism (CD) signal mostly due to the $\alpha$-helical secondary structure of HSA progressively decreased with the increase of the oxidative damage without showing a sigmoidal variation resembling the low-resolution structural parameters (Figures 4a and S5). The CD-based estimation of the secondary structure composition implied that the full oxidation-induced denaturation caused a loss of about $35 \%$ of the native $\alpha$-helical folding with advantage of the content of disordered structure.

The zeta potential measurements pointed out that the hypochlorite-induced chemical modification caused an increase of the protein's negative charge as soon as the OPR reached a critical level (Figure 4b). This threshold could be estimated
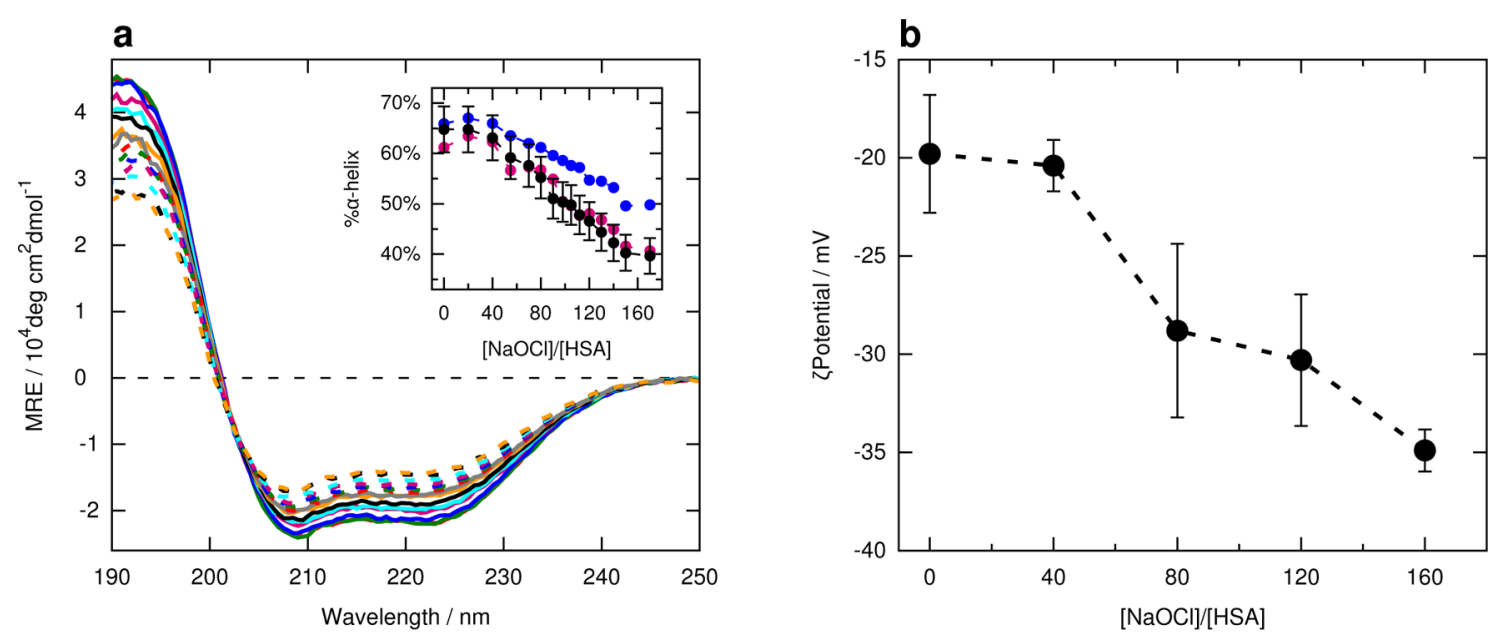

Figure 4. Results of complementary experiments aimed at assessing the protein secondary structure and surface charge variation induced by the oxidation process. (a) Far-UV CD spectra of oxidized HSA samples with oxidant/protein ratios ([NaOCl]/[HSA], OPR): 0 (red solid), 20 (green solid), 40 (blue solid), 55 (purple solid), 70 (cyan solid), 80 (black solid), 90 (orange solid), 98 (gray solid), 105 (red dotted), 112 (green dotted), 120 (blue dotted), 130 (purple dotted), 140 (cyan dotted), 150 (black dotted), 170 (orange dotted). In the inset, decrease of the $\alpha$-helical content estimated from the CD spectra as a function of OPR. The percentage of $\alpha$-helical structure estimated by means of the Chen equation ${ }^{28}$ (black dots), the BeStSel web server ${ }^{29}$ (purple dots), and the CONTIN/LL algorithm of the CDpro package ${ }^{49}$ (blue dots) are displayed. (b) Decrease of the zeta potential measured on oxidized HSA samples. 

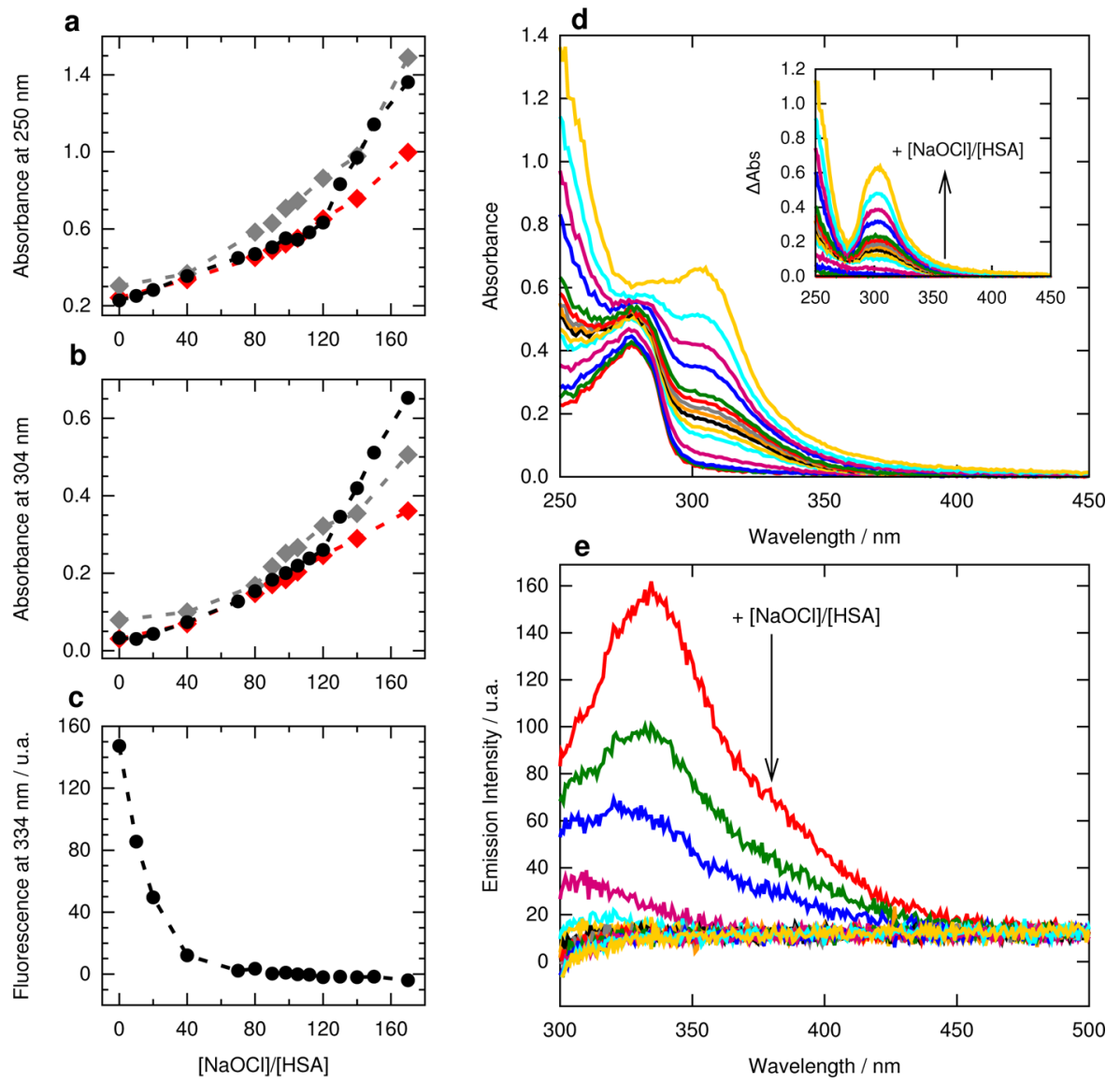

Figure 5. Variation of spectral features collected simultaneously with the SAXS data as a function of the oxidant/protein ratio ([NaOCl]/[HSA $]$ ). (a) UV absorbance at $304 \mathrm{~nm}$. (b) UV absorbance at $250 \mathrm{~nm}$. In these plots the absorbance data from the "single shot" experiments without SEC (black) are compared to those from the SEC experiment, both from the main peak (red) and oligomer peak (gray). The values from the SEC experiment are scaled according to the concentration estimated by the absorbance at $280 \mathrm{~nm}$ of the reference sample $([\mathrm{NaOCl}] /[\mathrm{HSA}]=0)$. $(\mathrm{c})$ Maximum fluorescence intensity. (d) UV-vis absorbance spectra of increasingly oxidized HSA. In the inset, the difference between UV-vis absorbance spectra of oxidized samples and the nonoxidized reference $([\mathrm{NaOCl}] /[\mathrm{HSA}]=0)$. (e) Fluorescence emission spectra with an excitation wavelength set at $293 \mathrm{~nm}$ and half width at half-maximum of $15 \mathrm{~nm}$. In (d) and (e) the color code for the increasing [NaOCl]/[HSA] values is 0 (red), 10 (green), 20 (blue), 40 (purple), 70 (cyan), 80 (yellow), 90 (black), 98 (orange), 105 (gray), 112 (red), 120 (green), 130 (blue), 140 (purple), 150 (cyan), 170 (yellow). The arrows represent a guide in order to follow the series of spectra in increasing order of oxidant/protein ratio.

around 80 in the OPR scale, similarly to the SAXS dimensional parameter variation.

The loss of roughly one-third of the $\alpha$-helical secondary structure could be probably attributable to the unfolding of the helices in the interdomain regions and in one of the three main lobes of the protein, which drifted apart. The intramolecular electrostatic repulsion generated with chemical modification could be responsible for the protein structural transition, determining a driving force to partial local disruption of the secondary structure and to the domain separation.

Spectral Features of Oxidized HSA. The use of the SUrF platform enabled us to collect UV/vis absorption and fluorescence emission spectra on the same sample volume as the SAXS data, thus allowing for a reliable correlation of the information derived from the three techniques (Figure 5). The UV spectra of the oxidized samples showed that a detectable chemical modification of the protein occurred for OPR values as low as 20 . According to the spectra obtained as a difference with respect to the curve of the nonoxidized sample, the main variations in the UV absorbance took place at about 250 and $304 \mathrm{~nm}$ (Figure 5d). The absorbance at these wavelengths was observed to almost linearly increase as a function of the OPR up to a value of 120 . For higher ratios, a second linear pattern with a larger slope was detected (black dots in Figure 5a,b) and this further increased absorbance at $304 \mathrm{~nm}$ was observed to decay in the 15 min time frame of the experiment (Supporting Information, Figure S3a,c). Interestingly, the changes observed in the UV spectra as a function of the OPR did not match the sigmoidal pattern of the SAXS parameters. The absorbance values maintained a linear increase in the range of oxidant doses which determined the abrupt structural expansion (OPR between 80 and 120). At higher doses, when the increased protein dimensions reached the plateau (OPR > 120), we noticed a steeper increase of the absorbance values due to the formation of labile species.

Similarly, there was no correlation between SAXS and the tryptophan maximum fluorescence emission. Instead, we found that the emission intensity very quickly decayed to less than $60 \%$ of the initial value for the lowest OPR value of the experiment $(\mathrm{OPR}=10)$. A complete loss of the tryptophan fluorescence was observed at OPR $=70$ where the SAXS data (reported for this sample in the Supporting Information, Figure S2) showed only slight deviations from the native protein, thus demonstrating that the chemical damage of HSA had to be severe in order to affect the protein conformation probed by SAXS. 

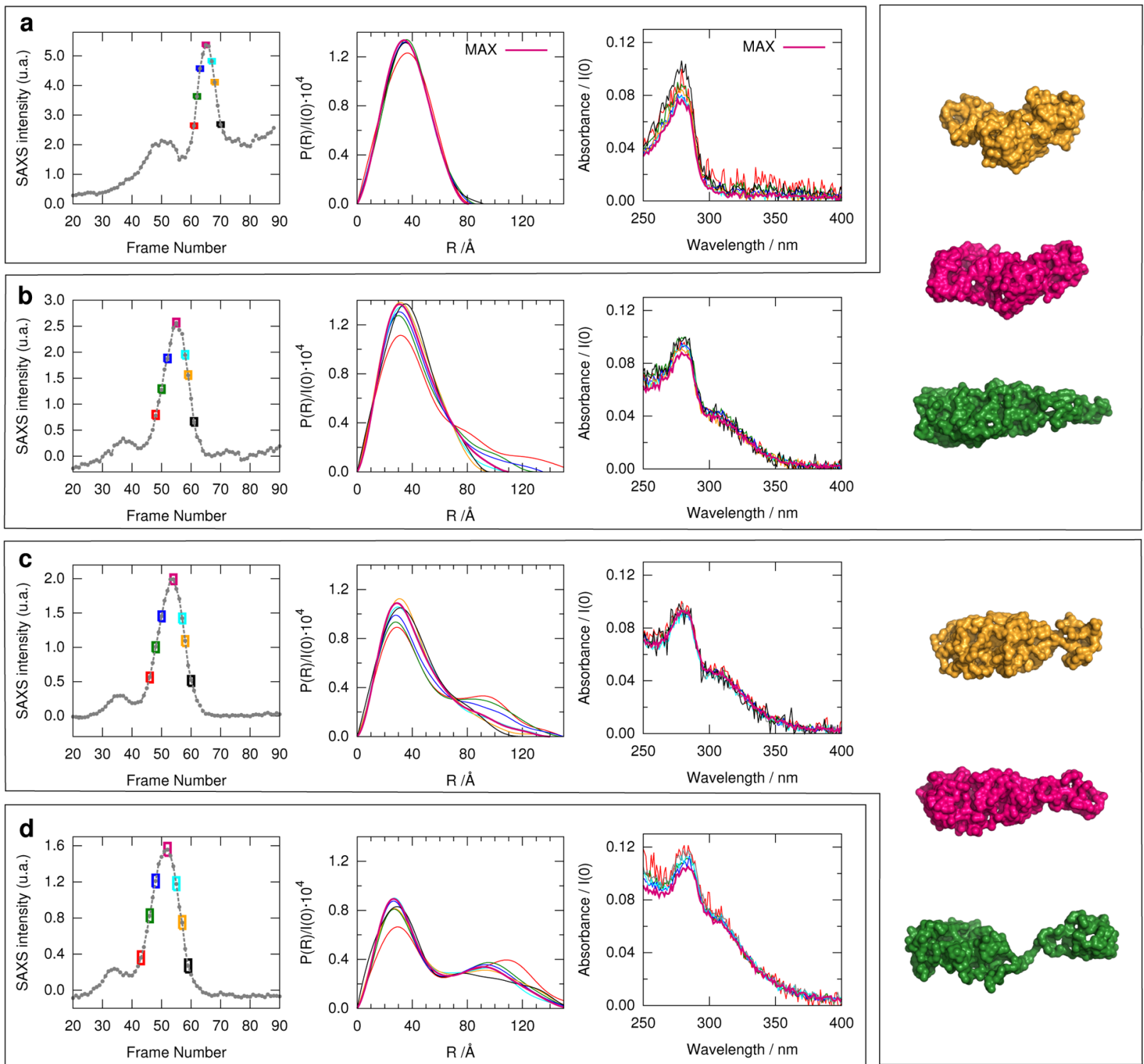

Figure 6. Comparison between the HSA monomer shapes derived from SAXS data collected at different points of the main SEC peak reveals different conformers can coexist in the intermediately oxidized samples. The results of this analysis for the samples with oxidant $/$ protein ratio $=0$ (reference, a), 90 (b), 105 (c), and 140 (d) are shown. In each case the chromatogram trace plotted using the buffer-subtracted scattered intensity integrated between 0.04 and $0.1 \AA^{-1}$ is displayed on the left. The $P(R)$ functions obtained for some of the experimental frames collected across the eluted peak are superimposed in the middle plot. In the third plot the UV spectra collected halfway during the SAXS exposure are also compared. The data are shown as solid lines of the same color as the box highlighting the corresponding frame. Both the $P(R)$ and the UV spectra plots were rescaled according to the $I(0)$ obtained from the $P(R)$. On the right some dummy-residue reconstructions obtained by fitting the SEC-SAXS data of the intermediately oxidized samples in $b$ and $c$ are shown, with a color code corresponding to the highlighted points in the chromatogram trace.

Oxidation of HSA Oligomers. The oxidation experiment on HSA performed using SEC in combination with the SUrF platform showed similar UV spectral features of the oxidized protein both in the main monomer peak and in the minor peak of oligomers (Supporting Information, Figure S6), indicating that the oligomeric forms present in the samples underwent chemical damage to a similar extent. Even if notably noisier, the SAXS data collected in correspondence with the secondary maximum observed at lower elution volumes than the monomer clearly showed that the average shape of the oligomeric species (mainly dimers) was also distinctly different in the oxidized protein as compared to the native form (Supporting Information, Figure S7). These findings suggested that the chemical modification did not induce more protein oligomerization but rather triggered the structural transition in the already existing oligomers. The characteristic bimodal $P(R)$ could be interpreted as indicative of dimers formed by protein molecules in the elongated form seen for the highly oxidized monomers.

Conformational Heterogeneity in the Intermediately Oxidized Samples. From the experimental SAXS data obtained in correspondence of the main SEC elution peak of the increasingly oxidized protein samples, two clearly distinguishable conformations could be pointed out, one typical of the native form (besides, stable even when the protein is oxidized with $40 \mathrm{~mol}$ equiv of hypochlorite) and the other one, markedly elongated and with bimodal $P(R)$, typical of samples treated with an oxidant amount higher than a critical level 
$(\geq 120)$. The low-resolution models recovered from the average SAXS data of the protein monomer at intermediate OPR values provided intermediate structures showing a progressive extension of a lobe from a globular moiety upon increasing oxidation (Figure 3). The correct interpretation of this gradual change of the average conformation between these two extremes could be debated as originating from the existence of intermediately elongated conformations at intermediate level of chemical damage or from the coexistence of better defined populations of different conformers.

A deeper exam of the protein conformation distribution could indeed be performed looking to the $P(R)$ functions obtained from data collected at different elution times within the SEC peak related to the monomer (see Figure 6). Relatively well-defined conformations were found in the native (OPR = $0)$ and fully oxidized $(\mathrm{OPR}=140)$ protein samples. The reference sample provided the typical bell shape of native HSA at all the elution volumes (Figure 6a) whereas the same characteristic bimodal shape of the $P(R)$ was found throughout the eluted peak of the highly oxidized sample at $\mathrm{OPR}=140$ (Figure 6d). In the case of two intermediately oxidized samples (Figure $6 \mathrm{~b}, \mathrm{c}$ ) a systematic variation toward more elongated forms was detected going from larger to smaller elution volumes (decreasing frame number). For the OPR $=90$ sample, the conformation with the smallest $D_{\max }$ could resemble the native form, while the form with larger $D_{\max }$ could be comparable with that present at the maximum of the OPR = 105 sample peak. On the lower volume side of this peak (Figure 6d), a gradual appearance of the characteristic bimodal $P(R)$ could be associated with the dumbbell shaped conformer found in the more oxidized samples as a minor contribution. By an average chemical point of view, the sampling of the UV/vis spectra showed no clear dishomogeneity in the oxidation level throughout the peak for each OPR value. In our opinion both an unresolved mixture of two main distinct conformations and more complex mixtures of a larger number of intermediates with oxidant ratio dependent fractions could explain the variation of the $P(R)$ functions throughout the peak of the intermediately oxidized samples. Chan et al. ${ }^{50}$ suggested that the most definitive determination of two-state behavior would be the experimental observation of two distinct populations near the midpoint of the transition and that, for slowly exchanging systems, this could be done by transport methods, such as SEC. In our experiment, we observed a notable peak broadening for the oxidized samples but not clearly distinct populations could be resolved.

\section{DISCUSSION}

The order of reactivity of the amino acids toward hypochlorite can guide an interpretation of the observed structural and spectral variations occurring at increasing oxidant doses, assuming that the hypochlorite equivalents are consumed to modify the protein's oxidizable residues in decreasing order of the kinetic constants for the reaction. Kinetics constants of the reactivity of amino acid model systems toward hypochlorite have been obtained by Pattison et al. ${ }^{12}$ and they were also used to explain the amount of amino acid consumption at different reactant levels for proteins with known sequence like BSA. ${ }^{13}$ In the case of BSA it was shown that the predictions based on the kinetic model, involving also secondary chloride transfer reactions from the chloramines generated, were essentially in agreement with the experimental composition of the oxidized protein after $24 \mathrm{~h}$ incubation, at least in terms of the average order of reactivity of the amino acids. Some discrepancies highlighted a role of the protein tertiary structure in modulating damage to specific residues.

Based on their results, one can build a rank of reactivity of the amino acids of HSA toward hypochlorite (Supporting Information, Table S1) which can be used to predict where their oxidation is expected on an OPR scale. The distribution of these potentially oxidizable amino acids in the HSA tertiary structure is also shown in the Supporting Information, Figure S8.

According to this order, tryptophan was thought to be oxidized at the low oxidant/protein molar ratio regime of our experiments ( OPR $<80$ ), as manifested by the complete depletion of tryptophan fluorescence in this range of oxidant dose.

The OPR critical region where the progressive opening toward an elongated conformation was detected is highlighted in yellow in Table S1. We notice that according to this order the lysine residues (abundant in HSA) should be the main target of the chemical modification in this regime of oxidant/ protein ratios. We could argue that these residues started a conversion to chloramines for molar ratios higher than $40 .^{48,51}$ After a critical molar excess (around 80), the modification of some pivotal lysine residues triggered the disruption of crucial interdomain salt-bridge interactions stabilizing the heart-shaped native form. Modification of additional lysine residues by higher hypochlorite doses (OPR between 80 and 120) would to a greater and greater extent stabilize an "open" conformation in which one of the end domains drifted apart from the rest of the oxidized protein. At OPR > 120, where no further opening was observed, a progressive oxidation of chloramines to dichloramines was believed to occur as demonstrated by the formation of an intense absorption band at $304 \mathrm{~nm}$ in the UV spectra. This band quickly decayed in time, as expected, due to the instability of the dichloramines. ${ }^{52}$ It is interesting to note that a further negative charge increase and helical structure disruption occurred in this regime (Figure 4).

Specific kinetic constants for the further conversion of the lysines from monochloramines to dichloramines were not measured, but the possibility of this conversion with an excess of hypochlorite was pointed out for the nitrogen-containing side-chains of glycosamines, ${ }^{53}$ and deduced for HSA in one of the early studies about hypochlorite modification. ${ }^{54}$

All together, we could deduce that the modification of electrostatic interactions through transformation of positively charged amine groups in unstable chloramines represented the major factor in determining protein native shape destabilization. Even if not evidenced as frequently as the more common methionine oxidation products, probably due to the difficulty in experimentally capturing the formation of still reactive chloramines by means of the most used chemical essays and mass spectrometric methods, ${ }^{55}$ lysine oxidation could be a relevant and general mechanism by which hypochloritedamaged proteins become unstable or undergo conformational changes. For example the correlation between the formation of protein chloramines and physiologically relevant functional variations induced by hypochlorite was demonstrated in the case of a protein acting as chaperone. ${ }^{56}$

\section{CONCLUSIONS}

The reported results highlight that HSA can tolerate the oxidation effect of large doses of hypochlorite in its folded state, as well as unfold only partially at extremely high oxidative 
damage, thus showing a tuned structural response. In addition, they show that the conformers are stable against aggregation and phase separation. A hypothesis suggested by this behavior would be that HSA can act as a biological "hypochlorite buffer" to control the local formation of this highly reactive oxidant. Indeed, these properties give the protein the capability to store a short-term oxidizing potential in the form of the reactive mono- and dichloramines and to release it in the environment upon diffusion. A direct consequence could be that HSA would have the ability to spread the bactericidal activity related to the hypochlorite-induced modifications and at the same time reduce the local oxidative burst and protect more susceptible proteins. The observed structural stability suggested that the biological functions of albumin could be partially preserved when oxidized.

Furthermore, the well-defined conformational variation of HSA triggered by the hypochlorite oxidation represents a new and fundamental addition to the structural rearrangements possible for this multidomain protein in response to functional $^{57}$ and denaturing conditions. ${ }^{2,46,58}$

For the several investigators working with albumin the reported biophysical characterization can be an important piece of information to rationalize experiments in the presence of oxidants. Meanwhile it opens the way for studies aimed at analyzing the effects of different conditions (such as the presence of small-molecule antioxidants or the binding of ligands) on the observed oxidation-induced structural transition. We could also speculate that the conformational change of HSA caused by the hypochlorite-induced chemical modification could help rationalize on a structural basis the observations about an apparent implication of the "chlorinated" form of HSA in specific receptor recognitions with potential roles in physiological processes like clearance of the damaged protein or inflammation stimulation. ${ }^{59-63}$

\section{ASSOCIATED CONTENT}

\section{S Supporting Information}

The Supporting Information is available free of charge on the ACS Publications website at DOI: 10.1021/acs.jpcb.6b08601.

Details on the experiments with the SUrF platform; additional results: combined rigid-body/dummy-residue modeling (Figure S1), SAXS analysis on the series of NaOCl-treated HSA samples not separated with SEC (Figure S2), time evolution of highly oxidized HSA (Figures S3, S4, and S5), oxidation of HSA oligomers (Figures S6 and S7); material referred to in the discussion: amino acids oxidizable by hypochlorite in HSA (Table S1 and Figure S8) (PDF)

\section{AUTHOR INFORMATION}

\section{Corresponding Authors}

*E-mail: alessandra.delgiudice@uniroma1.it.

*E-mail: nicolaeviorel.pavel@uniroma1.it; Telephone: (+39) 064991 3652; Fax: (+39) 06490631.

\section{ORCID ${ }^{\circ}$}

Alessandra Del Giudice: 0000-0002-1916-8300

Luciano Galantini: 0000-0001-5484-2658

Nicolae V. Pavel: 0000-0002-7637-4821

\section{Notes}

The authors declare no competing financial interest.

\section{ACKNOWLEDGMENTS}

The Unipharma Graduates Erasmus + project is acknowledged for providing economic support to A.D.G. during the visiting period in Lund, Sweden. The research leading to these results has received funding from the European Community's Seventh Framework Programme (FP7/2007-2013) CALIPSO under grant agreement $n^{\circ} 312284$. The MAX IV Laboratory is acknowledged for providing beamtime under the proposal 20140447. We are grateful to the I911 SAXS beamline staff for their support and to Prof. K. Schillén and Dr. S. Bayati for help during beamtime. We would like to thank Prof. P.O. Larsson for his advice about performing and interpreting SEC experiments and Prof. R. Samperi for the discussion about the SEC results.

\section{REFERENCES}

(1) Peters, T. J. All About Albumin; Elsevier, 1995.

(2) Fanali, G.; Di Masi, A.; Trezza, V.; Marino, M.; Fasano, M.; Ascenzi, P. Human Serum Albumin: From Bench to Bedside. Mol. Aspects Med. 2012, 33, 209-290.

(3) He, X. M.; Carter, D. C. Atomic Structure and Chemistry of Human Serum Albumin. Nature 1992, 358, 209-215.

(4) Anraku, M.; Yamasaki, K.; Maruyama, T.; Kragh-Hansen, U.; Otagiri, M. Effect of Oxidative Stress on the Structure and Function of Human Serum Albumin. Pharm. Res. 2001, 18, 632-639.

(5) Roche, M.; Rondeau, P.; Singh, N. R.; Tarnus, E.; Bourdon, E. The Antioxidant Properties of Serum Albumin. FEBS Lett. 2008, 582, $1783-1787$

(6) Bruschi, M.; Candiano, G.; Santucci, L.; Ghiggeri, G. M. Oxidized Albumin. The Long Way of a Protein of Uncertain Function. Biochim. Biophys. Acta, Gen. Subj. 2013, 1830, 5473-5479.

(7) Klebanoff, S. J. Oxygen Metabolites from Phagocytes. In Inflammation: Basic Principles and Clinical Correlates; Gallin, J. I., Snyderman, R., Eds.; Lippincott Williams and Wilkins: Philadelphia, 1999; pp 721-768.

(8) Bernofsky, C. Nucleotide Chloramines and Neutrophil-Mediated Cytotoxicity. FASEB J. 1991, 5, 295-300.

(9) Weiss, S. J. Tissue Destruction by Neutrophils. N. Engl. J. Med. 1989, 320, 365-376.

(10) Klebanoff, S. J. Myeloperoxidase: Friend and Foe. J. Leukocyte Biol. 2005, 77, 598-625.

(11) Winterbourn, C. C. Comparative Reactivities of Various Biological Compounds with Myeloperoxidase-Hydrogen PeroxideChloride, and Similarity of Oxidant to Hypochlorite. Biochim. Biophys. Acta, Gen. Subj. 1985, 840, 204-210.

(12) Pattison, D. I.; Davies, M. J. Absolute Rate Constants for the Reaction of Hypochlorous Acid with Protein Side Chains and Peptide Bonds. Chem. Res. Toxicol. 2001, 14, 1453-1464.

(13) Pattison, D. I.; Hawkins, C. L.; Davies, M. J. What Are the Plasma Targets of the Oxidant Hypochlorous Acid? A Kinetic Modeling Approach. Chem. Res. Toxicol. 2009, 22, 807-817.

(14) Colombo, G.; Clerici, M.; Giustarini, D.; Rossi, R.; Milzani, A.; Dalle-Donne, I. Redox Albuminomics: Oxidized Albumin in Human Diseases. Antioxid. Redox Signaling 2012, 17, 1515-1527.

(15) Temple, A.; Yen, T.-Y.; Gronert, S. Identification of Specific Protein Carbonylation Sites in Model Oxidations of Human Serum Albumin. J. Am. Soc. Mass Spectrom. 2006, 17, 1172-1180.

(16) Guedes, S.; Vitorino, R.; Domingues, R.; Amado, F.; Domingues, P. Oxidation of Bovine Serum Albumin: Identification of Oxidation Products and Structural Modifications. Rapid Commun. Mass Spectrom. 2009, 23, 2307-2315.

(17) Salavej, P.; Spalteholz, H.; Arnhold, J. Modification of Amino Acid Residues in Human Serum Albumin by Myeloperoxidase. Free Radical Biol. Med. 2006, 40, 516-525.

(18) Petrônio, M.; Fernandes, J. R.; de Menezes, M. L.; Ximenes, V. F. Oxidation of Bovine Albumin by Hypochlorous and Hypobromous 
Acids: Structural and Functional Alterations. Br. J. Pharm. Res. 2013, 3, 147-160.

(19) Lissi, E.; Alicia Biasutti, M.; Abuin, E.; León, L. A Fluorescence Study of Human Serum Albumin Binding Sites Modification by Hypochlorite. J. Photochem. Photobiol., B 2009, 94, 77-81.

(20) Azizova, O. A.; Aseychev, A. V.; Beckman, E. M.; Moskvina, S. N.; Skotnikova, O. I.; Smolina, N. V.; Gryzunov, Y. A.; Dobretsov, G. E. Studies of Oxidant-Induced Changes in Albumin Transport Function with a Fluorescent Probe K-35. Effect of Hypochlorite. Bull. Exp. Biol. Med. 2012, 152, 712-716.

(21) Wyatt, A. R.; Kumita, J. R.; Mifsud, R. W.; Gooden, C. a; Wilson, M. R.; Dobson, C. M. Hypochlorite-Induced Structural Modifications Enhance the Chaperone Activity of Human $\alpha 2$ Macroglobulin. Proc. Natl. Acad. Sci. U. S. A. 2014, 111, E2081-90.

(22) Haas, S.; Plivelic, T. S.; Dicko, C. Combined SAXS/UV-vis/ Raman as a Diagnostic and Structure Resolving Tool in Materials and Life Sciences Applications. J. Phys. Chem. B 2014, 118, 2264-2273.

(23) Pace, C. N.; Vajdos, F.; Fee, L.; Grimsley, G.; Gray, T. How to Measure and Predict the Molar Absorption Coefficient of a Protein. Protein Sci. 1995, 4, 2411-2423.

(24) Skou, S.; Gillilan, R. E.; Ando, N. Synchrotron-Based SmallAngle X-Ray Scattering of Proteins in Solution. Nat. Protoc. 2014, 9, 1727-1739.

(25) Petoukhov, M. V.; Billas, I. M. L.; Takacs, M.; Graewert, M. A.; Moras, D.; Svergun, D. I. Reconstruction of Quaternary Structure from X-Ray Scattering by Equilibrium Mixtures of Biological Macromolecules. Biochemistry 2013, 52, 6844-6855.

(26) Morris, J. C. The Acid Ionization Constant of $\mathrm{HOCl}$ from 5 to 35. J. Phys. Chem. 1966, 70, 3798-3805.

(27) Labrador, A.; Cerenius, Y.; Svensson, C.; Theodor, K.; Plivelic, T. The Yellow Mini-Hutch for SAXS Experiments at MAX IV Laboratory. J. Phys. Conf. Ser. 2013, 425, 72019.

(28) Chen, Y. H.; Yang, J. T.; Martinez, H. M. Determination of the Secondary Structures of Proteins by Circular Dichroism and Optical Rotatory Dispersion. Biochemistry 1972, 11, 4120-4131.

(29) Micsonai, A.; Wien, F.; Kernya, L.; Lee, Y.-H.; Goto, Y.; Réfrégiers, M.; Kardos, J. Accurate Secondary Structure Prediction and Fold Recognition for Circular Dichroism Spectroscopy. Proc. Natl. Acad. Sci. U. S. A. 2015, 112, E3095-103.

(30) Sreerama, N.; Woody, R. W. Estimation of Protein Secondary Structure from Circular Dichroism Spectra: Comparison of CONTIN, SELCON, and CDSSTR Methods with an Expanded Reference Set. Anal. Biochem. 2000, 287, 252-260.

(31) Nielsen, S. S.; Toft, K. N.; Snakenborg, D.; Jeppesen, M. G.; Jacobsen, J. K.; Vestergaard, B.; Kutter, J. P.; Arleth, L. BioXTAS RAW, a Software Program for High-Throughput Automated SmallAngle X-Ray Scattering Data Reduction and Preliminary Analysis. J. Appl. Crystallogr. 2009, 42, 959-964.

(32) Blanton, T. N.; Huang, T. C.; Toraya, H.; Hubbard, C. R.; Robie, S. B.; Louër, D.; Göbel, H. E.; Will, G.; Gilles, R.; Raftery, T. JCPDS-International Centre for Diffraction Data Round Robin Study of Silver Behenate. A Possible Low-Angle X-Ray Diffraction Calibration Standard. Powder Diffr. 1995, 10, 91-95.

(33) Orthaber, D.; Bergmann, A.; Glatter, O. SAXS Experiments on Absolute Scale with Kratky Systems Using Water as a Secondary Standard. J. Appl. Crystallogr. 2000, 33, 218-225.

(34) Guinier, A. La Diffraction Des Rayons X Aux Très Petits Angles: Application À L'étude Des Phénomènes Ultramicroscopiques. Ann. Phys. 1939, 12, 161-237.

(35) Petoukhov, M. V.; Franke, D.; Shkumatov, A. V.; Tria, G.; Kikhney, A. G.; Gajda, M.; Gorba, C.; Mertens, H. D. T.; Konarev, P. V.; Svergun, D. I. New Developments in the ATSAS Program Package for Small-Angle Scattering Data Analysis. J. Appl. Crystallogr. 2012, 45, 342-350.

(36) Svergun, D. I. Determination of the Regularization Parameter in Indirect- Transform Methods Using Perceptual Criteria. J. Appl. Crystallogr. 1992, 25, 495-503.

(37) Hansen, S. BayesApp: A Web Site for Indirect Transformation of Small-Angle Scattering Data. J. Appl. Crystallogr. 2012, 45, 566-567.
(38) Hansen, S. Simultaneous Estimation of the Form Factor and Structure Factor for Globular Particles in Small-Angle Scattering. J. Appl. Crystallogr. 2008, 41, 436-445.

(39) Svergun, D. I.; Petoukhov, M. V.; Koch, M. H. Determination of Domain Structure of Proteins from X-Ray Solution Scattering. Biophys. J. 2001, 80, 2946-2953.

(40) Volkov, V. V.; Svergun, D. I. Uniqueness of Ab Initio Shape Determination in Small-Angle Scattering. J. Appl. Crystallogr. 2003, 36, $860-864$.

(41) Kozin, M. B.; Svergun, D. I. Automated Matching of High- and Low-Resolution Structural Models. J. Appl. Crystallogr. 2001, 34, 3341.

(42) Petoukhov, M. V.; Svergun, D. I. Global Rigid Body Modeling of Macromolecular Complexes against Small-Angle Scattering Data. Biophys. J. 2005, 89, 1237-1250.

(43) Sugio, S.; Kashima, A.; Mochizuki, S.; Noda, M.; Kobayashi, K. Crystal Structure of Human Serum Albumin at 2.5 A Resolution. Protein Eng., Des. Sel. 1999, 12, 439-446.

(44) Uversky, V. N. Natively Unfolded Proteins: A Point Where Biology Waits for Physics. Protein Sci. 2002, 11, 739-756.

(45) Durand, D.; Vives, C.; Cannella, D.; Perez, J.; Pebay-Peyroula, E.; Vachette, P.; Fieschi, F. NADPH Oxidase Activator P67(phox) Behaves in Solution as a Multidomain Protein with Semi-Flexible Linkers. J. Struct. Biol. 2010, 169, 45-53.

(46) Leggio, C.; Galantini, L.; Konarev, P. V.; Pavel, N. V. UreaInduced Denaturation Process on Defatted Human Serum Albumin and in the Presence of Palmitic Acid. J. Phys. Chem. B 2009, 113, $12590-12602$.

(47) Del Giudice, A.; Leggio, C.; Balasco, N.; Galantini, L.; Pavel, N. V. Ibuprofen and Propofol Cobinding Effect on Human Serum Albumin Unfolding in Urea. J. Phys. Chem. B 2014, 118, 1004310051.

(48) Hawkins, C.; Davies, M. Hypochlorite-Induced Oxidation of Proteins in Plasma: Formation of Chloramines and Nitrogen-Centred Radicals and Their Role in Protein Fragmentation. Biochem. J. 1999, 548, 539-548.

(49) Sreerama, N.; Woody, R. W. Estimation of Protein Secondary Structure from Circular Dichroism Spectra: Comparison of CONTIN, SELCON, and CDSSTR Methods with an Expanded Reference Set. Anal. Biochem. 2000, 287, 252-260.

(50) Chan, H. S.; Bromberg, S.; Dill, K. A. Models of Cooperativity in Protein Folding. Philos. Trans. R. Soc., B 1995, 348, 61-70.

(51) Pattison, D. I.; Hawkins, C. L.; Davies, M. J. Hypochlorous Acid-Mediated Protein Oxidation: How Important Are Chloramine Transfer Reactions and Protein Tertiary Structure? Biochemistry 2007, 46, 9853-9864.

(52) Coker, M. S. A.; Hu, W.-P.; Senthilmohan, S. T.; Kettle, A. J. Pathways for the Decay of Organic Dichloramines and Liberation of Antimicrobial Chloramine Gases. Chem. Res. Toxicol. 2008, 21, 23342343.

(53) Pattison, D. I.; Davies, M. J. Reactions of MyeloperoxidaseDerived Oxidants with Biological Substrates: Gaining Chemical Insight into Human Inflammatory Diseases. Curr. Med. Chem. 2006, 13, $3271-3290$

(54) Arnhold, J.; Hammershmidt, S.; Wagner, M.; Mueller, S.; Arnold, K.; Grimm, E. On the Action of Hypochlorite on Human Serum-Albumin. Biomed. Biochim. Acta 1990, 49, 991-997.

(55) Raftery, M. J. Detection and Characterization of N-AlphaChloramines by Electrospray Tandem Mass Spectrometry. Anal. Biochem. 2007, 366, 218-227.

(56) Müller, A.; Langklotz, S.; Lupilova, N.; Kuhlmann, K.; Bandow, J. E.; Leichert, L. I. O. Activation of RidA Chaperone Function by NChlorination. Nat. Commun. 2014, 5, 5804.

(57) Ascenzi, P.; Fasano, M. Allostery in a Monomeric Protein: The Case of Human Serum Albumin. Biophys. Chem. 2010, 148, 16-22.

(58) Sadler, P. J.; Tucker, A. pH-Induced Structural Transitions of Bovine Serum Albumin. Histidine pKa Values and Unfolding of the NTerminus during the N to F Transition. Eur. J. Biochem. 1993, 212, 811-817. 
(59) Iwao, Y.; Anraku, M.; Hiraike, M.; Kawai, K.; Nakajou, K.; Kai, T.; Suenaga, A.; Otagiri, M. The Structural and Pharmacokinetic Properties of Oxidized Human Serum Albumin, Advanced Oxidation Protein Products (AOPP). Drug Metab. Pharmacokinet. 2006, 21, 140-146.

(60) Mikhalchik, E. V.; Smolina, N. V.; Astamirova, T. S.; Gorudko, I. V.; Grigorieva, D. V.; Ivanov, V. A.; Sokolov, A. V.; Kostevich, V. A.; Cherenkevich, S. N.; Panasenko, O. M. Human Serum Albumin Modified under Oxidative/halogenative Stress Enhances LuminolDependent Chemiluminescence of Human Neutrophils. Biophysics (Engl. Transl.) 2013, 58, 530-536.

(61) Binder, V.; Ljubojevic, S.; Haybaeck, J.; Holzer, M.; El-Gamal, D.; Schicho, R.; Pieske, B.; Heinemann, A.; Marsche, G. The Myeloperoxidase Product Hypochlorous Acid Generates Irreversible High-Density Lipoprotein Receptor Inhibitors. Arterioscler., Thromb., Vasc. Biol. 2013, 33, 1020-1027.

(62) Gorudko, I. V.; Grigorieva, D. V.; Shamova, E. V.; Kostevich, V. A.; Sokolov, A. V.; Mikhalchik, E. V.; Cherenkevich, S. N.; Arnhold, J.; Panasenko, O. M. Hypohalous Acid-Modified Human Serum Albumin Induces Neutrophil NADPH Oxidase Activation, Degranulation, and Shape Change. Free Radical Biol. Med. 2014, 68, 326-334.

(63) Pasterk, L.; Lemesch, S.; Leber, B.; Trieb, M.; Curcic, S.; Stadlbauer, V.; Schuligoi, R.; Schicho, R.; Heinemann, A.; Marsche, G. Oxidized Plasma Albumin Promotes Platelet-Endothelial Crosstalk and Endothelial Tissue Factor Expression. Sci. Rep. 2016, 6, 22104. 